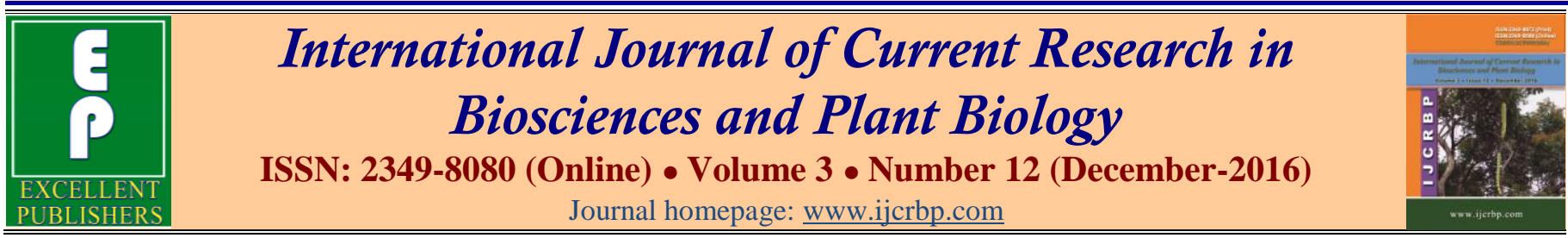

\title{
Acute Toxicity Evaluation of Environmental Hormones Using Daphnia magna
}

\author{
William Chung and Jongbin Lee*
}

Provert Research Laboratory, 111 Charlotte Place Ste\#10o, Englewood Cliffs, NJ o7632, Unites States.

*Corresponding author.

\begin{tabular}{|c|c|}
\hline Abstract & Article Info \\
\hline \multirow{17}{*}{$\begin{array}{l}\text { Daphnia magna are common planktonic crustacean of the subclass Phyllopoda that can } \\
\text { be found in lakes and ponds all over the Holarctic. They have been the subject of } \\
\text { biological research since the } 1700 \text { s because of their value as an experimental organism; } \\
\text { this is due to their plentitude, easily observable organs, easy maintenance, and quick } \\
\text { reproduction rate. As a result, they are a well-known reference organism to study } \\
\text { aquatic environmental contamination. Using this species, three different environmental } \\
\text { hormones, deltamethrin, bifenthrin, and glyphosate, were introduced to develop an } \\
\text { environmental pollution research model. The standard method of determining } \\
\text { physiological differences of Daphnia magna typically observes only its heartbeat. } \\
\text { However, in this study, one more variable was added in order to more accurately } \\
\text { reflect the physiological changes underwent by Daphnia magna when their } \\
\text { environmental conditions were altered: their swimming rate. In the actual experiment, } \\
\text { the Daphnia magna were exposed to a serial dilution solution of the environmental } \\
\text { hormones in a contamination model and then had their heartbeat rate measured. The } \\
\text { experiment was then repeated under the same conditions for estimating their change in } \\
\text { swimming rate. In each pollution model technique, the data demonstrated a } \\
\text { mathematical relation of heartbeat rate and swimming rate with polynomial } \\
\text { functionality. The sensitivity along the dilution concentration was different between } \\
\text { the \% heartbeat change and \% swimming rate change, which might mean that both } \\
\text { parameters should be mutually complementary and carried out simultaneously, when } \\
\text { any environmental hormone's toxicity is required to be thoroughly studied. The } \\
\text { dangers of environmental hormones should not be downplayed and a more effective } \\
\text { method for checking acute toxicity should be useful. }\end{array}$} & $\begin{array}{l}\text { Accepted: } 24 \text { November } 2016 \\
\text { Available Online: } 06 \text { December } 2016\end{array}$ \\
\hline & Keywords \\
\hline & Acute toxicity \\
\hline & Endocrine distrupters \\
\hline & Environmental hormones \\
\hline & $\begin{array}{l}\text { Heartbeat change } \\
\text { Swimming rate }\end{array}$ \\
\hline & \\
\hline & \\
\hline & \\
\hline & \\
\hline & \\
\hline & \\
\hline & \\
\hline & \\
\hline & \\
\hline & \\
\hline & \\
\hline
\end{tabular}

\section{Introduction}

The endocrine system consists of multiple glands secreting hormones that regulate various body functions of reproduction, sexual activities, metabolism, growth and development, tissue function and mood, to say the least (Vandenberg et al., 2012). Environmental hormones disturb the integrity of the endocrine system. Many chemical compounds act as endocrine disruptors, such as insecticides, herbicides, plasticizers. If these chemical compounds spread outside their area of intended use, they can stay in the environment and flow down into local bodies of water and wreak havoc upon ecosystems in the area (Folke et al., 2004). 
Environmental hormones (EH), often known as endocrine disruptors, are harmful synthetic chemicals that can prevent the body from functioning properly by acting as hormones. Therefore, the continuous accumulation of $\mathrm{EH}$ can trigger many catastrophic damages to our ecological systems inconspicuously hidden to us (Pereira et al., 1996).

Daphnia are an important aquatic organism as a first consumer that lives in lakes and rivers (Wetzel, 1995). Because of the characteristics of the species, it serves as a reference species to determine the level of the environmental contamination (Nebeker et al., 1984). They have a very favorable physiological character: a transparent body, allowing researchers to monitor their hearts' palpitations. When Daphnia encounter drastic changes in their environment, their heartbeat rate, and consequentially their motility, reflect those changes (Gregory, 2008). Therefore, understanding the acute effects on the cardiac function and phototactic swimming rate should be of significance (Biesinger and Christensen, 1972). Among many studies, a relationship between change in heartbeat rate and exposure to chemical compounds has been established (Lovern et al., 2007; Lawson, 2001; Dzialowski et al., 2006; VillegasNavarro, et al., 2003). However, not much has been investigated in regard to swimming rate.

Swimming rate is one of the variables typically used to describe swimming behavior by the effects of environmental conditions. However, a comparison of a number of parameters is needed to make sure we are using the appropriate parameters to describe any toxicity underlying complicate physiology. The hypothesis of this study was that the change in swimming rate should have some functional relationship with the perturbation of heartbeat rate of Daphnia as an indication of the acute toxic effects of EH. In this study, the change in Daphnia's heartbeat after a 30-minute incubation was investigated to find correlation with change in swimming rate induced by the phototaxis after exposure to the chemical compounds. The three mentioned EH models each have distinct characteristics in chemical structures and functional modalities.

Deltamethrin, [(S)-cyano-(3-phenoxyphenyl) methyl] (1R,3R)-3-(2,2-dibromoethenyl)-2,2-

dimethylcyclopropane-1-carboxylate (C22H19Br2NO3) is a synthetic pyrethroid which, in its unadulterated state, lacks both color and odor (Decourtye et al., 2004). Pyrethroids are synthetic organic compounds derived from pyrethrins, naturally occurring chemical compounds with insecticidal applications found in pyrethrum (Casida, 1980). Pyrethrum is an insecticide made from the oleoresin extract obtained from dehydrated flower heads of Chrysanthemum cinerariifolium as well as Chrysanthemum coccineum, both plants are belonged to the genus Pyrethrum, a genus of several Old World plants, now Chrysanthemum or Tenacetum. Six distinct chemical compounds contribute to pyrethrum's insecticidal properties; these are known collectively as the pyrethrins. The ketoalcoholic esters present in pyrethrin are the source of its insecticidal characteristics as insects are highly vulnerable to their strongly lipophilic properties and subsequently have their nervous systems paralyzed (Casida and Quistad, 1998). Despite being fatal to many household pests such as spiders, fleas, ticks, carpenter ants, carpenter bees, cockroaches, and bed bugs, pyrethrins are largely harmless to mammals as they are not readily absorbed through the skin. However, it is still a neurotoxin and will thus temporarily attack the nervous system when it contacts skin, causing reddening of the skin, numbness, burning, and paresthesia (Ledoux, 2011). Synthetic pyrethroids, such as deltamethrin, are, in essence, "meaner" versions of pyrethrins, as they were more toxic to both insects and mammals and have much longer break down times, leading to more extended periods of contamination if exposed to the ecosystems. As there are no antidotes to deltamethrin poisoning, treatment is purely symptomatic, and standard procedure entails allowing the body to metabolize until any and all remaining toxicity has left the body (Marrs, 1993). Despite the potential for poisoning, deltamethrin products have become globally popular due to their proficiency in exterminating insects (Naylor and Ehrlich, 1997). It experiences widespread use in urban and residential areas for garden variety pest extermination as well as vector control of certain strains of malaria. Due to its significant presence, deltamethrin's sphere of influence has a large radius (Cooper and Dobson, 2007). While it does not pose any insurmountable threat to humans, household animals, and other mammals, and birds, deltamethrin is lethal to fish and is potentially devastating to underwater ecosystems.

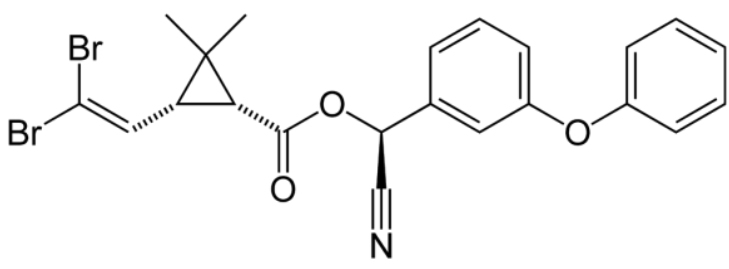

Fig. 1: The chemical structure of deltamethrin compound. 
Deltamethrin (Fig. 1) products are one of the most popular and widely being consumed insecticides globally and have shifted very popularly with pest control operators and individuals in the United States. This material is a member of one of the safest classes of pesticides: artificial pyrethroids. This pesticide is highly poisonous to aquatic life, especially fish, and must be used with extreme caution around water. Although considered safe to use around humans, it is still neurotoxic to humans.

Bifenthrin, 2-Methyl-3-phenylphenyl) methyl (1S,3S)-3[(Z)-2-chloro-3,3,3-trifluoroprop-1-enyl]$2,2-$ dimethylcyclopropane-1-carboxylate, is a white, waxy solid and has a slight sweet smell (Jiang et al., 2004). Being a synthetic pyrethroid as well, it shares many properties with deltamethrin. Its primary use is combating infestations of red fire ants by attacking the nervous system of these insects. It is similar to deltamethrin in all its properties, including its lethality to insects and aquatic life, but relative harmlessness to humans and other mammals and birds. Bifenthrin is highly toxic to bees as well. Although it may be itching, burning, and general irritation, bifenthrin poses no real threat to humans (WHO Pesticide Evaluation Scheme, 2003). It has been tentatively labeled as a possible carcinogen, but laboratory testing found no conclusive connection between bifenthrin exposure and cancer. The true threat posed by bifenthrin is the potential havoc it could wreck upon ecosystems if significant amounts leak into the environment.

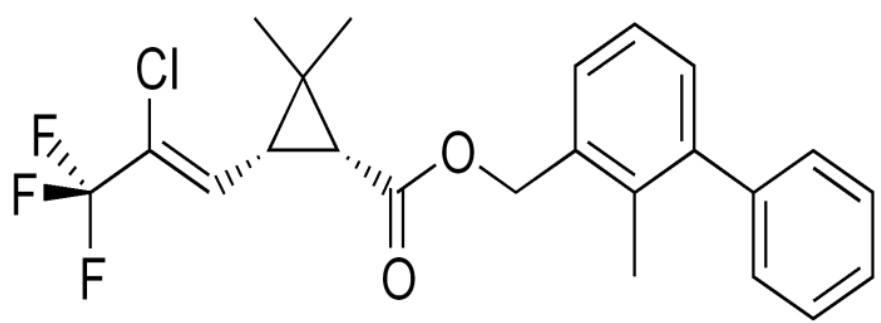

Fig. 2: The image of the chemical compound, Bifenthrin.

Bifenthrin (Fig. 2) is hardly soluble in water, so nearly all bifenthrin will stay in the sediment, but it is very toxic to the aquatic life. Even in small concentrations, fish and other water animals are harmed by bifenthrin. One of the reasons for the high responsiveness of water animals is that they have a slow metabolism. Therefore, bifenthrin will stay longer in the system of the aquatic animals. Another reason for the high sensitivity of fish is the effect of bifenthrin as ATPase-inhibitor. The water animals need ATP to control the osmotic balance of oxygen. If the aquatic animal is no longer capable of taking up oxygen because ATP can no longer be applied, the aquatic animals will die.

N-(Phosphonomethyl) glycine; 1071-83-6; Glyphosphate; Glycine, N-(phosphonomethyl), also known as glyphosate, ( $\mathrm{N}$-(phosphonomethyl) glycine, is unlike the former two chemicals in that it is neither a pyrethroid nor an insecticide; it is an organophosphorus compound with herbicidal and crop desiccative applications (Duke and Powles, 2008). It finds widespread use in weed elimination, especially in annual broadleaf weeds and grasses that compete with crops (Johnson et al., 2009). It is usually absorbed through the leaves of the plant, although sometimes through the roots as well, and then proceeds to inhibit plant growth by halting the production of tyrosine, tryptophan, and phenylalanine (amino acids evolved in synthesis. Its herbicidal properties were discovered by John Franz in 1970 while working with Monsanto in 1970 (Aoki, 2004). It would go on to be commercially introduced in 1974 under the trade name Roundup (Aoki, 2004). This effectiveness in eliminating unwanted plants paired with the development of Roundup Ready crops (an alliterated epithet given to crops genetically engineered to tolerate the effects of Roundup) would cause glyphosate to go on to be immensely popular among farmers and civilians alike, finding usage in over 160 countries with over 1.4 billion pounds of the product used per year. Farmers quickly adopted glyphosate, especially soon after Monsanto commercialized the glyphosate-resistant Roundup Ready crops, farmers have applied glyphosate even more. The resistant Roundups facilitate farmers to remove weeds without sacrificing their crops (Duke and Powles, 2008).

In 2007, the glyphosate was sprayed the most for removing weeds in the United States. Further, it was ranked the second place consumed for residential area, and for industrial purposes. Its usage has been expected in 2016 for the application frequency and amounts of glyphosate-based herbicides. That is, the global emergence and spread of glyphosate-resistant weeds will be continued for the time being (Myers et al., 2016). However, this popularity would go on to make its carcinogenic properties all the more horrifying. Studies conducted discovered that high-dose exposure of glyphosate was documented to have reproductive and developmental effects. Although glyphosate is not highly toxic to fish and aquatic life, it can still affect them by killing the plants that offer nutrients and shelter. 


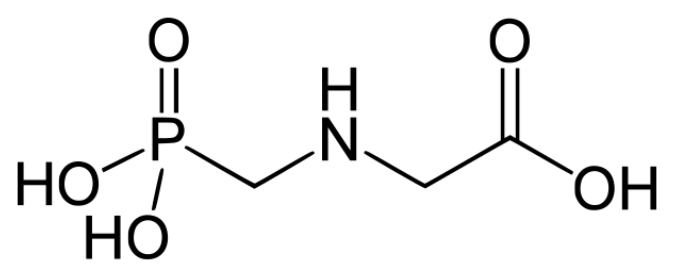

Fig. 3: The chemical structure of glyphosate.

The chemical compound, glyphosate (Fig. 3) was chosen, since they were the EHs that were being used by the most all around the world. In addition, it should be a great idea to compare the molecular structures of the environmental hormones to see any common element of inducing acute toxicity.

This study might be substantial in the light of quantifying the relationship between heartbeat change and swimming rate under the influence of the environmental hormones of deltamethrin, bifenthrin, and glyphosate selected to be investigated. If a relationship between swimming rate and exposure to environmental hormones is discovered, it could be very useful for understanding any toxic effects from the testing environmental hormones.

\section{Materials and methods}

\section{Materials and reagents}

A compound microscope $(\times 400)$ was purchased from Home Science Tools (Billings, MT, USA). Daphnia magna were obtained from the Carolina Biological Supplies (Burlington, NC, USA), and maintained in a ten-gallon fish tank with the diet of active yeast and tropical fish flakes until used. For the assembly of the swimming rate chamber, Plexiglas acrylic plastic panel was purchased from the ULine (Breinigsville, PA). Other materials such as slide glass, test tubes, test tube racks, and rulers, were readily available and used accordingly. Energy-saving fluorescent light bulbGE60W was purchased from 1000 Bulbs (Garland, TX) and three-way Switches were acquired from Parts Tree (Austin, TX); these were required to create the chamber. Deltamethrin was purchased from Jansen Distributing (Spokane, WA, USA).

Bifenthrin was purchased from Quali-Pro (Raleigh, NC, USA), and Glyphosate was purchased from Monsanto (St Louis, MO, USA). Disposable gloves and safety goggles were purchased from CVS (Fort Lee, NJ, USA) and utilized as personal protective equipment according to the regulation of biomedical laboratory.

\section{Assembly of swimming rate chamber}

In this study, a phototactic testing system was assembled using a clear plastic tube (3.75" O.D. $\times 23.5^{\prime \prime}$ long) and black-painted Plexiglas rectangular box $\left(24^{\prime \prime} \times 8^{\prime \prime} \times 8^{\prime \prime}\right)$ with two fluorescent bulbs, one situated at the top and the other at the bottom, controlled by a three-way switch (Fig.4). The plastic tube was marked by permanent marker at one inch intervals from bottom to top so that the Daphnia's location at any given time could be recorded with ease. Daphnia positions were recorded at every 30 seconds. Screening was performed with a flashlight in order to find active Daphnia that generally moved toward light. Then, five of those Daphnia were placed into the plastic tube, which had been filled with $750 \mathrm{~mL}$ of culturing water was filled in advance. The plastic tube was then moved into the rectangular box. The two bulbs were activated individually at 3 minute intervals and the Daphnia swam accordingly. The positions of the Daphnia were recorded every 30 seconds and averaged out. The data gathered here would later be used as a comparison to the average positions every 30 seconds in solutions containing $\mathrm{EH}$.

In another set of studies, 30-minute incubation study was carried out. Six serially diluted solutions of testing chemicals were prepared (Fig. 4). The Daphnia's heartbeats were measured before and after the incubation. The differences in heartbeats between preand post-incubation were calculated on a coordinate with dilution factors; their relationship was determined by the slope of linear regression analysis. This study would help us understand clearly on the relation of heartbeat and swimming rate under the influence of EHs. Three EHs were purchased from local stores such as deltamethrin, bifenthrin, and glyphosate. They were sold in diluted solution $(0.04 \% \sim 5 \%)$, and were used sparingly as little as possible in the range of $200 \mathrm{uL}$ to $1 \mathrm{~mL}$ for each.

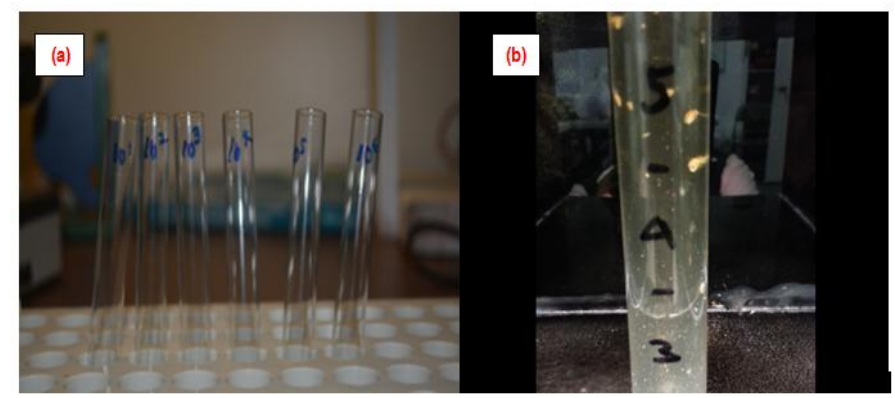

Fig. 4: (a) The test tubes were labeled and filled with serially diluted solutions of EHs. (b) A part of the swimming tube from which the location of swimming Daphnia was read and recorded accordingly. 


\section{Heartbeat counting methods}

The standard laboratory procedure for determining the heartbeats per minute figure (BPM) of a Daphnia specimen is a clunky process. It requires two people in order to be performed properly and the difference in reaction times between the two people causes an intrinsic discrepancy in data. Instead of needing one person count the heartbeats of the Daphnia and the other watch the stopwatch, it is possible for the entire procedure to be performed by a single person by changing the independent variable from time to heartbeats. Instead of measuring how many times the heartbeats in 10 seconds, one measures the number of seconds required for 40 heartbeats. All that is needed then is to divide 40 by the time recorded and then multiply the result by 60 to arrive at a BPM figure.

\section{Incubation solution preparation}

Six test tubes were picked up and labeled on the surface as $10^{1} / 0,10^{2} / 5,10^{3} / 10,10^{4} / 15,10^{5} / 20$ and $10^{6} / 25$. A Daphnia was incubated in each tube 30 minutes. The number before the slash was the dilution factor and the numbers after the slash represents the number of minutes after the start of the incubation that a Daphnia was placed into that tube. After labeling, each test tube was filled with $1800 \mathrm{uL}$ of nutrient water which was obtained from a culturing tank with a $1000 \mathrm{uL}$ Eppendorf pipette. A serial dilution was then performed with each of the environmental hormones. The testing EH of $200 \mathrm{~mL}$ was transported using a pipette into the first test tube of $10^{1} / 0$. The mixture was thoroughly shaken and mixed. Then, $200 \mathrm{uL}$ of the first diluted solution in $10^{1} / 0$ was obtained and transported into the second test tube of $10^{2} / 5$. This same serial dilution and mixing procedures was performed up to the last test tube of $10^{6} / 25$.

\section{Incubation method}

After the preparation of the serially diluted incubation solutions described above, a Daphnia was picked from the container, and moved onto a slide glass to obtain a pre-incubation BPM measurement. It was then dropped into the first test tube and the incubation stopwatch was started. After every 5 minutes another Daphnia was dropped into the next test tube. At 30 minutes of the incubation stopwatch, the Daphnia that had been incubated for 30 minutes was taken out from the first tube $10^{1} / 0$ and its heartbeat was counted for its postincubation heartbeat and recorded. This process was repeated for each of the remaining Daphnia. The difference in BPM before and after incubation was then plotted against the dilution factor.

\section{Results and discussion}

\section{Population sampling}

The heartbeat of Daphnia has been known to be varied due to the effects of temperature, body size, and environmental conditions. In order to minimize variance in data, the heartbeat rates of Daphnia in relation to their sizes was evaluated during a preliminary study on what size of Daphnia would be suitable for our research. Three beakers were prepared with labels of small size (1.0 $\mathrm{mm}$ or less), medium size (larger than $1.0 \mathrm{~mm}$, and smaller than $2 \mathrm{~mm}$ ), and large size (greater than $2 \mathrm{~mm}$ ). A compound microscope was used to measure the Daphnia. After the Daphnia were measured, they were separated into their respective beakers. As seen in the in Fig. 5, the Daphnia of medium size displayed the most stable heartbeat. The Daphnia of different sizes had heartbeats that were either too fast or slow and would result inerratic data. In this study, exclusively medium sized Daphnia were used for all remaining parts of the experiment based on this preliminary set of study.

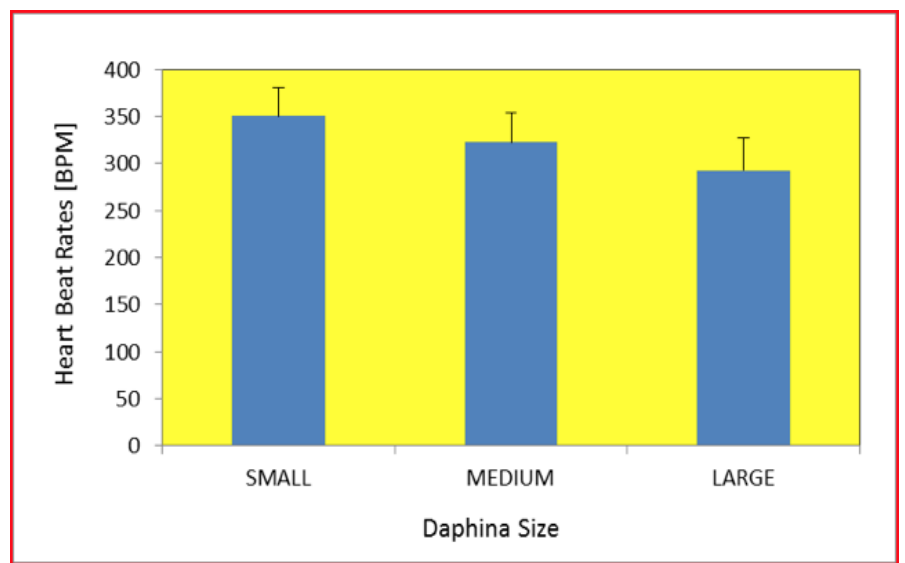

Fig. 5: The graph shows the apparent tendencies of the heartbeat by the sizes of the Daphnia magna $(\mathrm{N}=20)$. The small size represented $1 \mathrm{~mm}$ or less Daphnia magna. The medium size represented the species with larger than $1.0 \mathrm{~mm}$ but smaller than $2.0 \mathrm{~mm}$. The large size was the entities with the size over $2.0 \mathrm{~mm}$.

\section{Heartbeat change in nutrient water}

The Daphnia were subjected to the 30-minute incubation study in the nutrient water, the culturing water obtained from a Daphnia tank, in order to conduct a negative control. As expected, only minor change was recorded, which could have come from the 
measuring variability and conditions. The data allowed us to confirm that our experimental technique did not produce any noticeable disturbance from the serial dilution and incubation duration (Fig. 6). Further, the minimal influence from the background variability was understood further.

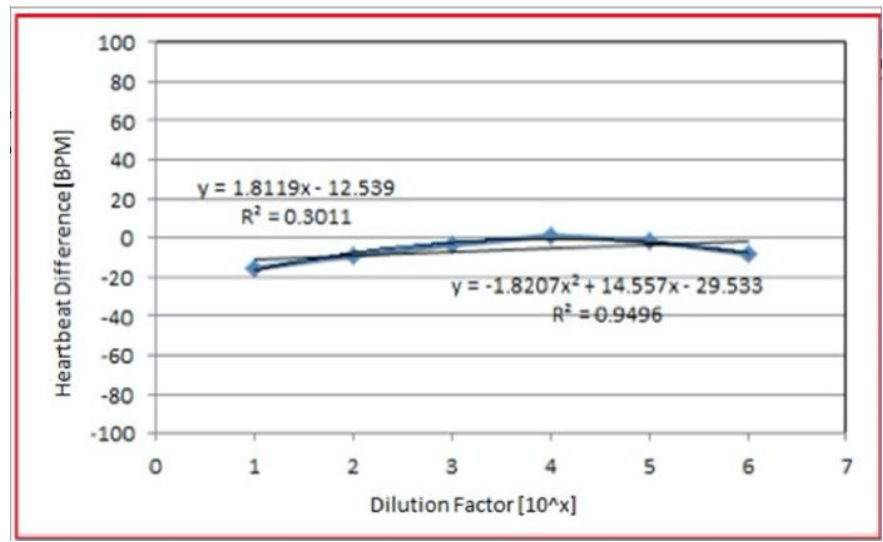

Fig. 6: The change of heartbeat in the nutrient water was presented on the graph. This worked as a negative control of the study to see the differentiated effect of the EHs in the followed experiments (mean, $\mathrm{N}=4$ ).

\section{Heartbeat change in alcohol solution}

Ethyl alcohol was used to see the change in heartbeat after 30 minutes of incubation in serially diluted solution. The alcohol solution was chosen to be a positive control, since it has been studied from other groups of scientists and known to cause much change in heartbeat for the incubation study. As seen in contrast to the negative control, the slope of heartbeat-dilution plot was significantly higher than that of the negative control to be 42.909 [BPM/Dilution Factor] (Fig. 7). Through this data, it was understood that the chemical compounds disturb the heartbeat from which other physiological effects could be subsequently triggered in the end.

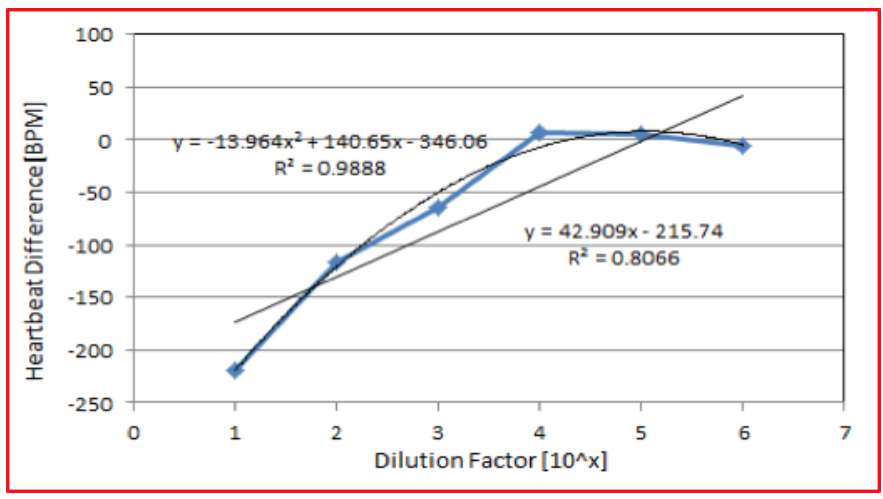

Fig. 7: The change of heartbeat of Daphnia in alcohol.
Compared to the negative control data, the heartbeat tendency was changed drastically. Interestingly, at the point of dilution $1 / 10^{5}$, the heartbeat change was slightly slowed to become a normal environment's heartbeat performance. Even though the rates were different, the other chemicals also showed the similar patterns (mean, $\mathrm{N}=4$ ).

\section{Heartbeat change in deltamethrin solutions}

Fig. 8 shows the relation of heartbeat difference before and after the 30-minute incubation in serially diluted deltamethrin solution. As expected, the heartbeat change was decreased with respect to the increase of dilution factor with high slope of 72.58 [BPM/Dilution Factor].

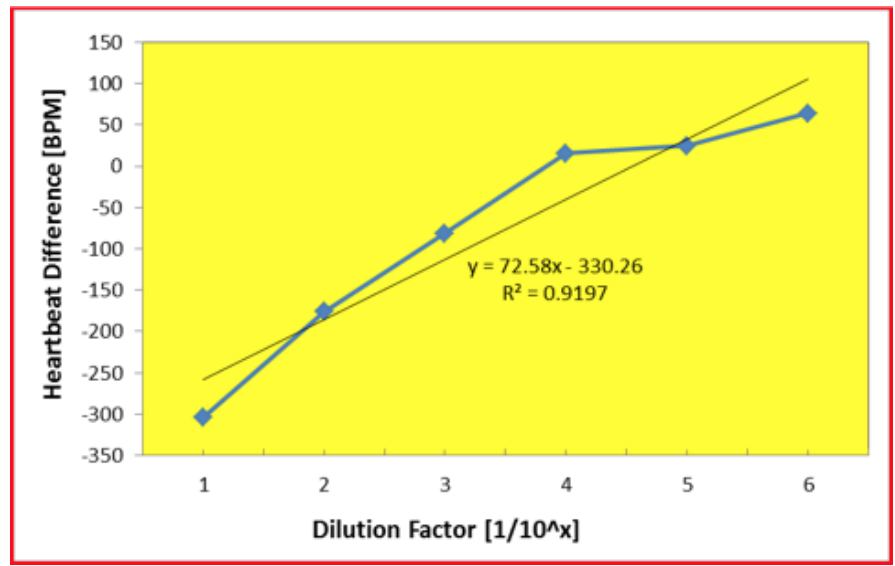

Fig. 8: The change of heartbeat in Daphnia from deltamethrin diluted solution.

Fig. 8 shows that the high concentration of deltamethrin affected Daphnia's heartbeat significantly. Also, lowered concentration of deltamethrin affected Daphnia's cardiac performance not that significantly. Interestingly, at the point of dilution factor $1 / 10^{5}$, the heartbeat changes were near to nothing to become a normal heartbeat performance. Even though the rates were different, the heartbeat changes from other chemicals also showed the typical patterns (mean, $\mathrm{N}=4$ ).

\section{Heartbeat change in bifenthrin solutions}

Fig. 9 shows the slope of bifenthrin's effect on the species' physiological performance. The slope of 38.292 [BPM/Dilution Factor] was smaller than that of deltamethrin. However, their trend of decreasing with respect to the increase in dilution factor was similar. Due to showing typical pattern of the data, it could mean that the experimental method may be appropriate for this type of study $(\mathrm{N}=4)$. 


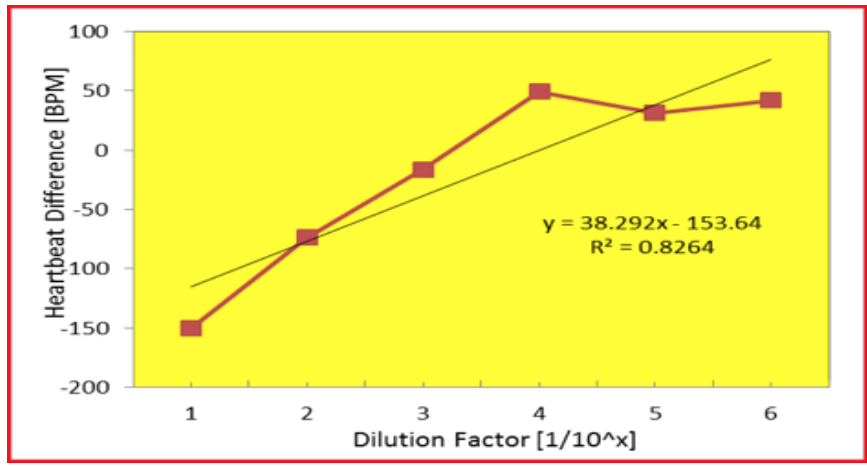

Fig. 9: The heartbeat difference in bifenthrin diluted solutions was plotted with respect to the dilution factor.

The data in Fig. 9 suggests the high concentration of bifenthrin induced significant heartbeat change. When lowered its concentration, Daphnia's cardiac performance was not affected that amount. At the dilution factor $1 / 10^{5}$, the heartbeat changes were minimal to restore closely to the normal heartbeat performance. The heartbeat changing trend was similar to that of other chemical compounds.

\section{Heartbeat change in glyphosate solutions}

The slope of glyphosate, 51.637 [BPM/Dilution Factor] was smaller than that of deltamethrin. However, the trend of decreasing with respect to the increase in dilution factor was similar. The similarity of the profiles might be the general characteristic relations of heartbeat difference and dilution factor.

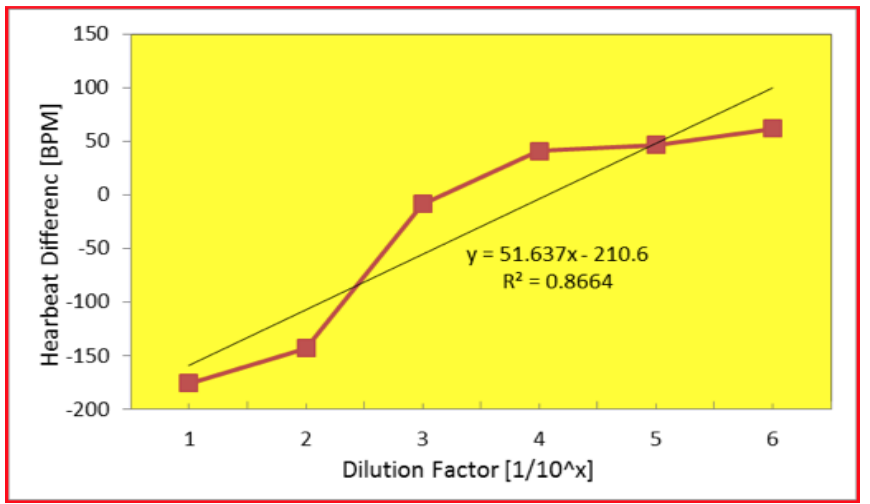

Fig. 10: The change of heartbeat in glyphosate diluted water was presented in the graph.

Fig. 10 demonstrates that the high concentration of glyphosate affected Daphnia's heartbeat significantly as other chemicals did. And, when it lowered concentration of glyphosate, Daphnia's cardiac performance was proportionally followed. At dilution factor $1 / 10^{5}$, the heartbeat was close to the normal heartbeat. Even though the rates were different, the heartbeat change from other chemicals showed the similar patterns.

\section{Swimming rate in deltamethrin solutions}
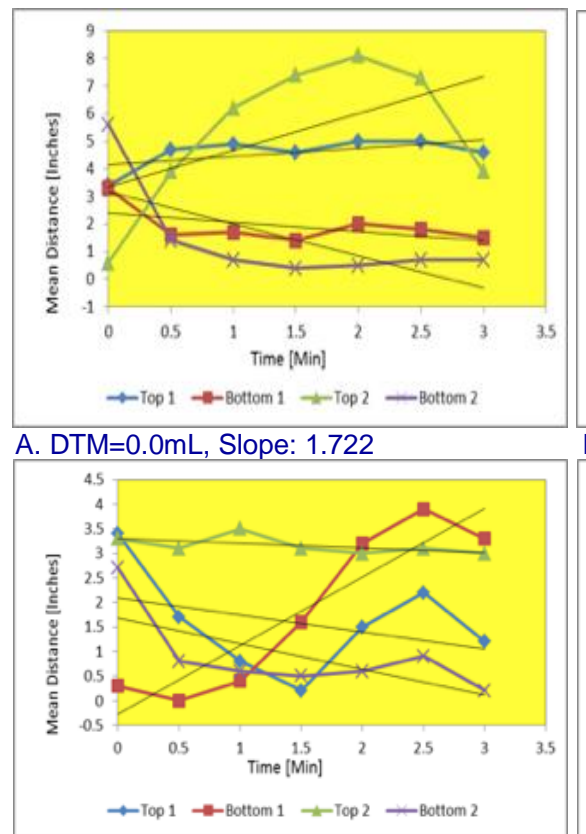

D. $\mathrm{DTM}=0.3 \mathrm{~mL}$ (1/2500), Slope $=0.332$
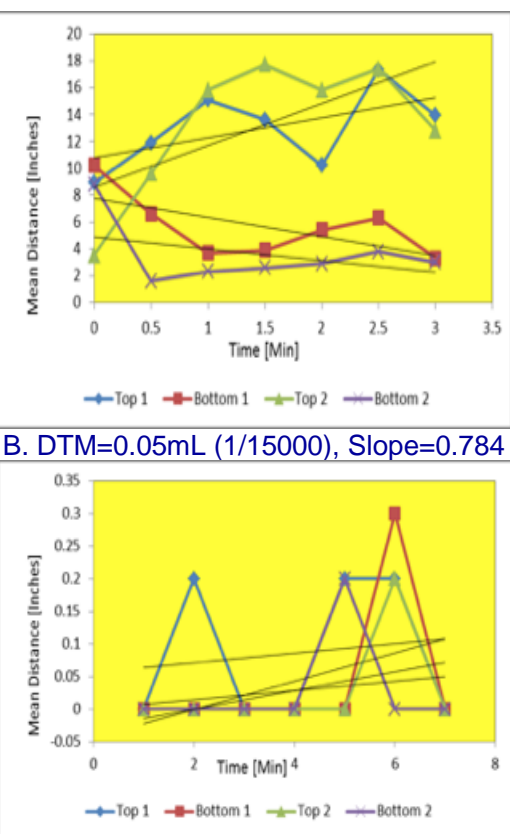

E. DTM=0.5mL (1/1500), Slope $=0.05$
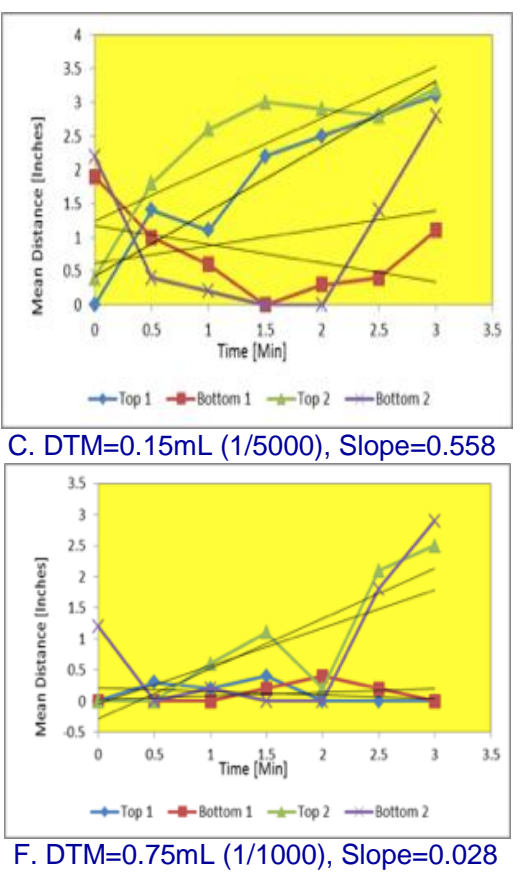

Fig. 11: The graphs provide the changes of the mean location of Daphnia with time, while the concentration of the EH increasing. In this case, the EH was deltamethrin. From the left top, to right bottom, the amount of deltamethrin added to the tube increased.Top1 stands for light on first at the top, then, bottom 1 for light on at the bottom and so on. 
In Fig. 11, six graphs were provided to help clarify the experimental system. For each concentration of deltamethrin, the same process was carried out. The positions of the daphnia were monitored at every 30 seconds and averaged out. By adding into the column, their mean location for each concentration was charted and plotted on their respective graphs. The mean location curves were linearized in an Excel worksheet and the slopes of the regression line for each trial were averaged. The average procedure was carried out using the lightoriented relative slopes. For examples, when the slope was positive toward light, its slope was defined to be positive, while the slope was negative, when the slope was negative toward light. When the bottom light was on, and the slope of swimming line was negative, its sign for averaging was changed to be positive. Briefly, the sign of slope was phototactically-oriented. The light was on and off twice alternatively. The four slopes were averaged arithmetically. The graphs demonstrated that as concentration in the swimming column increased, the mean slopes approached zero.

\section{Swimming rate in bifenthrin solution}

In Fig. 12, six graphs provided were to clarify the experimental system. The same process used in the deltamethrin swimming rate tests was utilized. The positions of the daphnia were monitored at every 30 seconds and averaged out. Their movement for each concentration was charted and plotted on their respective graphs. The movement curves were linearized and the slopes of the linearization for each trial were averaged. The graphs clearly demonstrate that as concentration increased, the slopes approached zero.
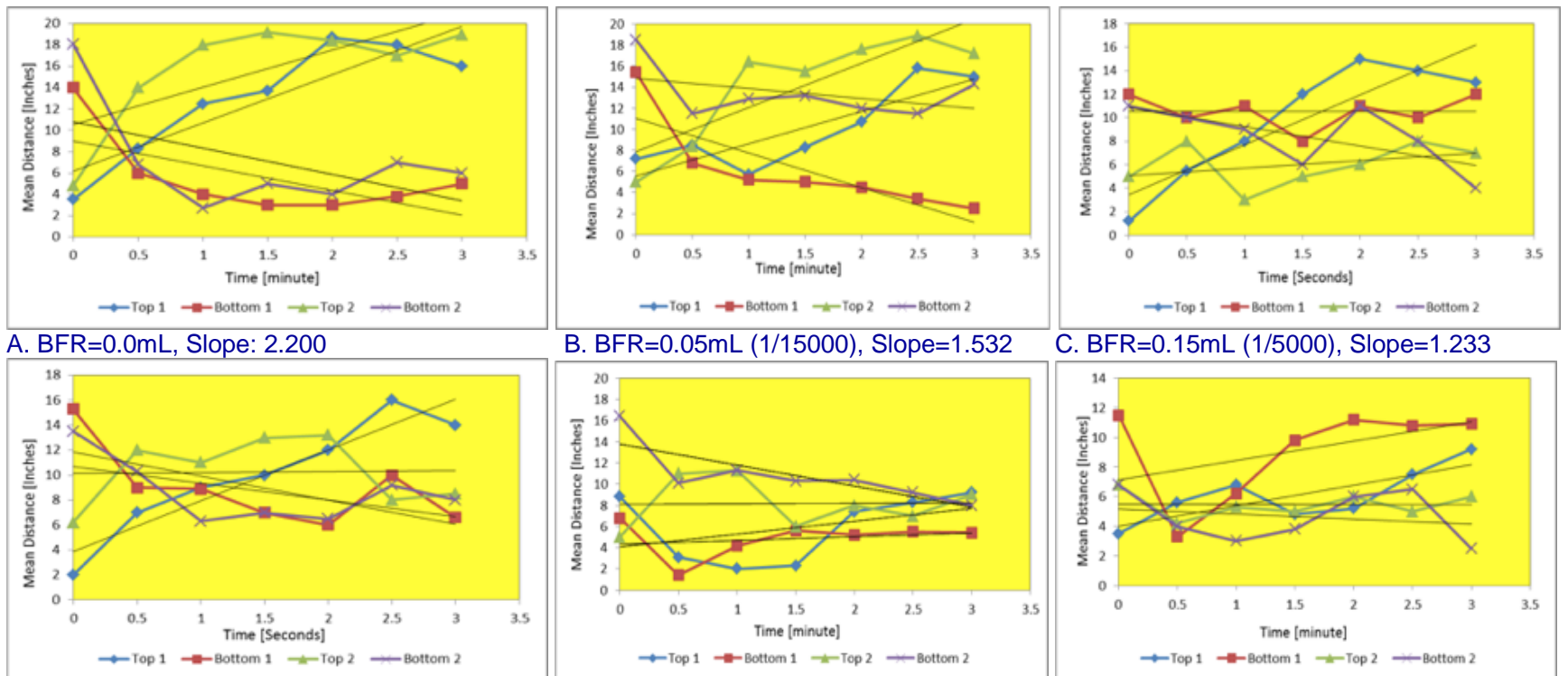

D. $B F R=0.45 \mathrm{~mL}$ (1/1700), Slope: 1.722
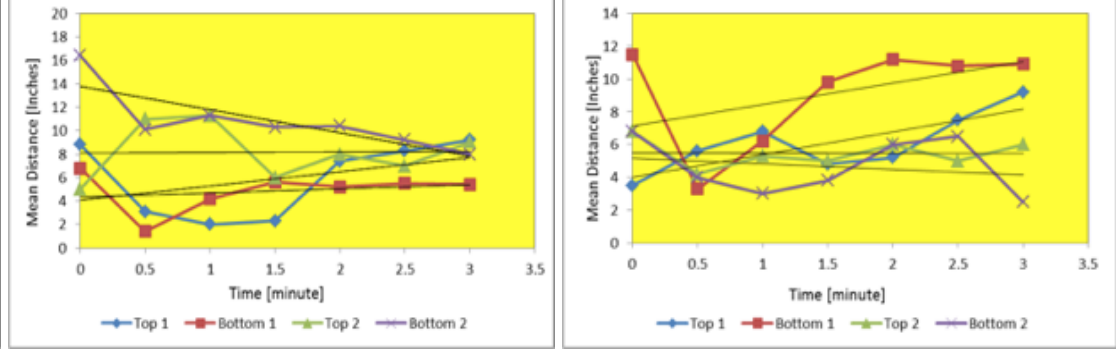

Fig. 12: The graphs illustrate the changes of the mean location of Daphnia with time, while the concentration of the EH increasing. In this case, the EH was bifenthrin. From the left top, to right bottom, the amount of bifenthrin added to the tube increased. Top1 stands for light on first at the top, then, bottom 1 for light on at the bottom and so on.

\section{Swimming rate in glyphosate solution}

In Fig. 13, the six graphs were provided to clarify the experimental system. The positions of the daphnia were monitored at every 30 seconds and averaged out. Their movement for each concentration was charted and plotted on their respective graphs.

The movement curves were linearized and the slopes of the linearization for each trial were averaged. The graphs clearly demonstrate that as concentration increased, the slopes approached zero. When comparing how the slopes changed over six concentrations for all three environmental hormones, they all follow the same trend; the slope being roughly two in the first graph and almost zero in the sixth. However, when comparing just the sixth graphs of each $\mathrm{EH}$, the average heights of the zeroed slopes were different. The average height for the highest concentration of deltamethrin was nearly zero while the average height for the highest concentration of bifenthrin was approximately five. This data could be useful when studying the effects of $\mathrm{EH}$ on the swimming patterns. 

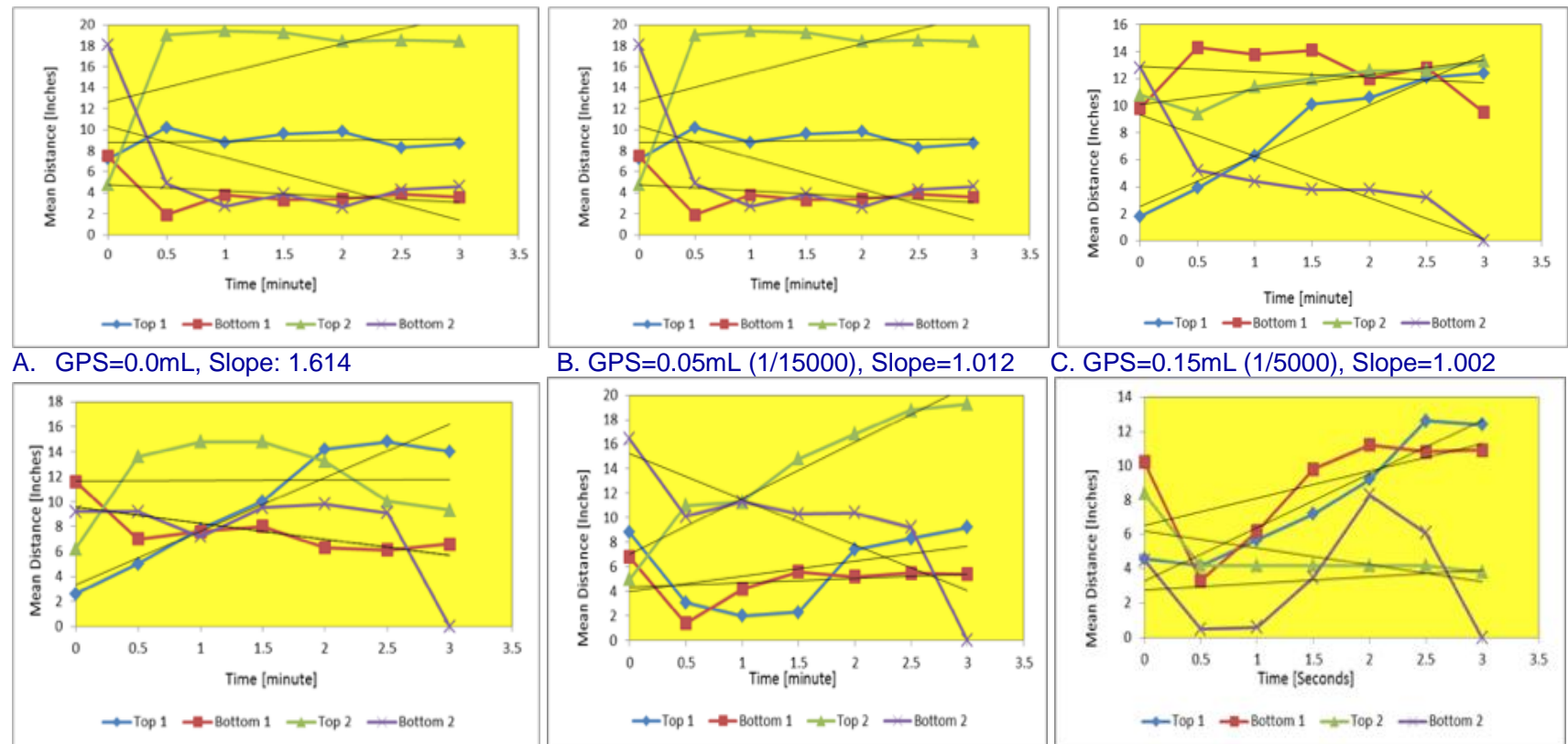

C. GPS $=0.15 \mathrm{~mL}(1 / 5000)$, Slope $=1.002$
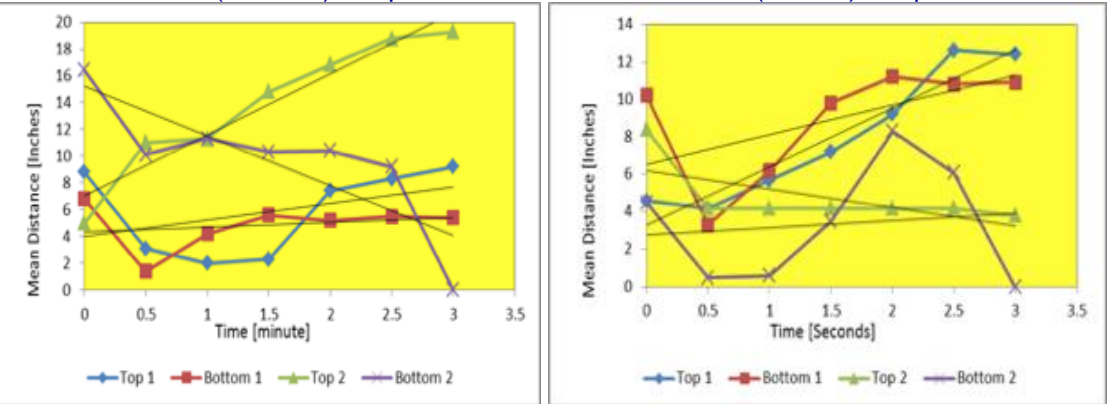

D. GPS $=0.30 \mathrm{~mL}(1 / 2500)$, Slope: 0.857

E. GPS $=0.50 \mathrm{~mL}(1 / 1500)$, Slope $=0.842$

F. DTM $=0.75 \mathrm{~mL}(1 / 1000)$, Slope $=0.043$

Fig. 13: The graphs show the changes of the mean location of Daphnia with time, while the concentration of the EH increasing in the swimming tube in glyphosate solution. From the left top, to right bottom, the amount of glyphosate was increasingly added to the tube. Top1 stands for light on first at the top, then, bottom 1 for light on at the bottom and so on.

\section{Relationship of heartbeat and swimming rate}

The added amount of EH into the swimming column was converted into the dilution factor by dividing the volume of nutrient water by the amount of $\mathrm{EH}$ in order to compare with the percent change of heartbeat at the equivalent amount of the EH solution. Based on the comparison of the numbers at $15,000\left(1: 1.5 \times 10^{4}\right)$ dilution solutions, the mean slope percent change in swimming rate for deltamethrin, bifenthrin, and glyphosate was $54.7 \%, 30 \%$, and $36 \%$. However, the percent change in heartbeat rate was $4 \%, 16 \%, 14 \%$ for deltamethrin, bifenthrin, and glyphosate, respectively. The $\%$ heartbeat change was more linearly decreased, when the EH was higher concentration. The sensitivity which was the slope of the graph was changed accordingly. On the other hand, the \%swimming change was more linearly decreased, when the EH was relatively low concentration. That was, it could be inferred that the daphnia's swimming ability was disappeared earlier than the heart palpitation, if the EH kept adding to a swimming tube as seen from the data. A critical dilution concentration might be existed for choosing which technique should be appropriate, or both techniques should be performed at the same time to see a big picture of acute toxicity as MRI and PET scans are both needed and mutually supplementary for diagnosing a brain cancer accurately. This study could lead us to a conclusion that both techniques might be mutually supplementary and should be carried out simultaneously, if a comprehensive understanding on acute toxicity of any EH was required. As can be seen in Fig. 14, the relationship of percent change in swimming rate plotted against percent change in heartbeat rate can be show as the polynomial graph $\mathrm{y}=21.7 x^{2}+83.8 x-26.1$ at 15,000 $\left(1: 1.5 \times 10^{4}\right)$ dilution solutions. However, this relation could not be not reflected all across the dilution solutions tested in this study.

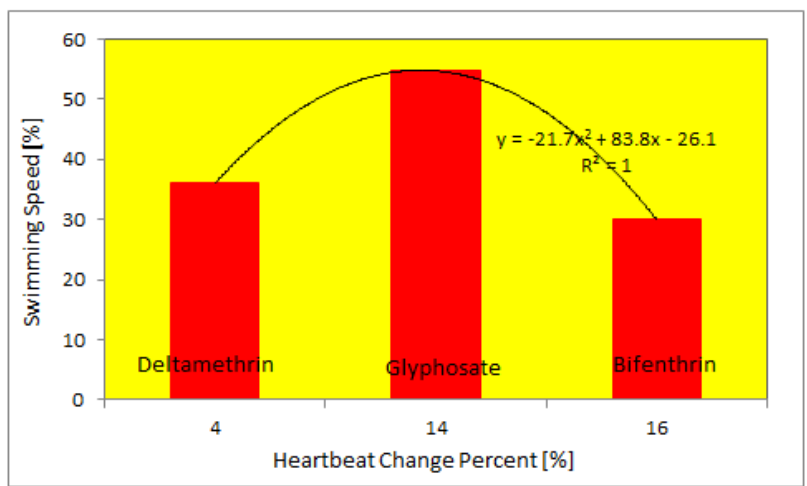

Fig. 14: The comparison of the three different EHs effect on the physiological performance of Daphnia magna at $1: 1.5 / 10^{4}$ dilution. Swimming rate \%change was smaller in bifenthrin, deltamethrin and glyphosate in order, while heartbeat change percent was smaller in deltamathrin, glyphosate and bifenthrin in order. 


\section{Conclusion}

The study was conducted to compare the change in heartbeat with the swimming rate which was presented as the slope of displacement changes with time. When compared at 15,000 dilution solution of the three EHs, the $\%$ swimming rate change was greater than the \% heartbeat change in each EH solution. And, there existed a polynomial functional relationship among the environmental hormones between the \% change of heartbeat and swimming rate. The sensitivity range along the EH concentration was different between the \% heartbeat change and \% swimming rate change, which might mean that both parameters should be mutually considered, when any environmental hormone's toxicity is studied. For further study, other groups of environmental hormones might be examined if the data we obtained here could show any general tendency for other chemical compounds.

\section{Conflict of interest statement}

Authors declare that they have no conflict of interest.

\section{References}

Aoki, K., 2004. Malthus, Mendel, and Monsanto: Intellectual property and the law and politics of global food supply: An introduction. J. Envtl. Law. Litig. 19, 397.

Biesinger, K. E., Christensen, G. M., 1972. Effects of various metals on survival, growth, reproduction, and metabolism of Daphnia magna. J. Fish. Board Canada. 29(12), 16911700 .

Casida, J. E., 1980. Pyrethrum flowers and pyrethroid insecticides. Environ. Health Perspect. 34, 189.

Casida, J. E., Quistad, G. B., 1998. Golden age of insecticide research: Past, present, or future? Annu. Rev. Entomol. 43(1), 1-16.

Cooper, J., Dobson, H., 2007. The benefits of pesticides to mankind and the environment. Crop Prot. 26(9), 13371348.

Decourtye, A., Devillers, J., Cluzeau, S., Charreton, M., PhamDelègue, M. H., 2004. Effects of imidacloprid and deltamethrin on associative learning in honeybees under semi-field and laboratory conditions. Ecotoxicol. Environ. Saf. 57(3), 410-419.

Duke, S. O., Powles, S. B., 2008. Glyphosate: A once-in-a-century herbicide. Pest Manag. Sci. 64(4), 319325.

Dzialowski, E. M., Turner, P. K., Brooks, B. W., 2006. Physiological and reproductive effects of beta adrenergic receptor antagonists in Daphnia magna. Arch. Environ. Contam. Toxicol. 50(4), 503-510.

Folke, C., Carpenter, S., Walker, B., Scheffer, M., Elmqvist,
T., Gunderson, L., Holling, C. S., 2004. Regime shifts, resilience, and biodiversity in ecosystem management. Annu. Rev. Ecol. Evol. Syst. 35, 557-581.

Gregory, N. G., 2008. Physiology and Behaviour of Animal Suffering. John Wiley \& Sons.

Jiang, W., Kon, R. T., Othoudt, R. A., Leavitt, R. A., Kumar, S., Geissel, L. D., Gomaa, E. A., 2004. Method development, validation, and analysis of bifenthrin residues in fresh and dry cilantro foliages and cilantro seeds using GC-ECD. Bull. Environ. Contam. Toxicol. 73(1), 9-16.

Johnson, W. G., Davis, V. M., Kruger, G. R., Weller, S. C., 2009. Influence of glyphosate-resistant cropping systems on weed species shifts and glyphosate-resistant weed populations. Eur. J. Agron. 31(3), 162-172.

Lawson, A. E., 2001. Using the learning cycle to teach biology concepts and reasoning patterns. J. Biol. Educ. 35(4), 165169.

Ledoux, D., 2011. Pest Control Simplified for Everyone: Kill, Repel, or Mitigate Pests with or without Pesticides. Universal-Publishers.

Lovern, S. B., Strickler, J. R., Klaper, R., 2007. Behavioral and physiological changes in Daphnia magna when exposed to nanoparticle suspensions (titanium dioxide, nano-C60, and $\mathrm{C} 60 \mathrm{HxC} 70 \mathrm{Hx}$ ). Environ. Sci. Technol. 41(12), 44654470 .

Marrs, T. C., 1993. Insecticides in urban environment. In: Proceedings of the First International Conference on Urban Pests (eds.: Wildey, K.B., Robinson, Wm. H.). S. 9-13.

Myers, J. P., Antoniou, M. N., Blumberg, B., Carroll, L., Colborn, T., Everett, L. G., Vandenberg, L. N., 2016. Concerns over use of glyphosate-based herbicides and risks associated with exposures: A consensus statement. Environ. Health, 15(1), 1.

Naylor, R., Ehrlich, P. R., 1997. Natural pest control services and agriculture. In: Nature's Services: Societal Dependence on Natural Ecosystems (Ed.: Daily, G. C.), Island Press, Washington, DC. pp.151-174.

Nebeker, A. V., Cairns, M. A., Gakstatter, J. H., Malueg, K. W., Schuytema, G. S., Krawczyk, D. F., 1984. Biological methods for determining toxicity of contaminated freshwater sediments to invertebrates. Environ. Toxicol. Chem. 3(4), 617-630.

Pereira, W. E., Domagalski, J. L., Hostettler, F. D., Brown, L. R., Rapp, J. B., 1996. Occurrence and accumulation of pesticides and organic contaminants in river sediment, water and clam tissues from the San Joaquin River and tributaries, California. Environ. Toxicol. Chem. 15(2), 172-180.

Vandenberg, L. N., Colborn, T., Hayes, T. B., Heindel, J. J., Jacobs Jr, D. R., Lee, D. H., Zoeller, R. T., 2012. Hormones and endocrine-disrupting chemicals: low-dose effects and nonmonotonic dose responses. Endocrine Rev. 33(3), 378-455.

Villegas-Navarro, A., Rosas-L, E., Reyes, J. L., 2003. The heart of Daphnia magna: Effects of four cardioactive 
drugs. Comp. Biochem. Physiol. Part C: Toxicol. WHO Pesticide Evaluation Scheme, 2003. WHO: WHO Pharmacol. 136(2), 127-134.

Pesticide Evaluation Scheme (WHOPES)-

Wetzel, R. G., 1995. Death, detritus, and energy flow in WHO/CDS/WHOPES/2002.5. World Health Organization aquatic ecosystems. Freshwater Biol. 33(1), 83-89. (WHOPES), Geneva.

\section{How to cite this article:}

Chung, W., Lee, J., 2016. / Acute toxicity evaluation of environmental hormones using Daphnia magna. Int. J.

Curr. Res. Biosci. Plant Biol. 3(12), 53-63. doi: http://dx.doi.org/10.20546/ijcrbp.2016.312.008 